\title{
Manejo ortodóncico-periodontal de recesión gingival
}

\author{
Orthodontic-periodontal Management of Gingival Recession \\ Constanza Urtubia Manríquez ${ }^{1 a}$, Cristóbal García Izquierdo ${ }^{2 b}$, Carolina Alarcón Azócar ${ }^{10}$ \\ (1D) https://orcid.org/0000-0002-8050-9303 (D) https://orcid.org/0000-0002-3311-3808 (D) https://orcid.org/0000-0001-7506-1076
}

\section{Resumen}

Restaurar la posición dentaria y la salud periodontal es esencial en ortodoncia y periodoncia. Los objetivos de este artículo fueron evaluar un caso clínico mediante el análisis de la relación de los movimientos dentales y las recesiones gingivales, determinar el momento oportuno para la realización de la cirugía mucogingival y nombrar las posibles etiologías del movimiento dental indeseado a causa de un retenedor fijo. Presentamos un informe de 30 meses de seguimiento de una paciente, cuya queja principal fue sobre un cambio progresivo en la posición dental asociado con una recesión gingival, a pesar de un retenedor fijo de un tratamiento ortodóncico previo. Se indicó retratamiento de ortodoncia y cirugía periodontal para cubrir el defecto por recesión. Luego del tratamiento, se concluyó que los movimientos dentales pueden desencadenar, agravar o mejorar las recesiones gingivales, por lo que es importante una evaluación previa individual. El momento de la cirugía mucogingival depende de la dirección del movimiento, los tejidos circundantes y el fenotipo periodontal del paciente. torque

Palabras claves: cirugía bucal, ortodoncia, recesión gingival, retenedores ortodóncicos,

\begin{abstract}
Restoring dental position and periodontal health is essential in orthodontics and periodontics. The objectives of this article were to evaluate a clinical case by analyzing the relationship of tooth movements and gingival recessions, to determine the opportune moment for performing mucogingival surgery, and to name the possible etiologies of unwanted tooth movement due to a fixed retainer. We present a 30-month follow-up report of a patient, whose main complaint was of a progressive change in dental position associated with a gingival recession, despite a fixed retainer from previous orthodontic treatment. Orthodontic retreatment and periodontal surgery were indicated to cover the defect due to recession. After treatment, it was concluded that tooth movements can trigger, aggravate or improve gingival recessions, which is why a prior individual evaluation is important. The timing of mucogingival surgery depends on the direction of movement, surrounding tissues, and the patient's periodontal phenotype.
\end{abstract}

Keywords: oral surgery,orthodontics, gingival recession, orthodontic retainers, torque

\section{Introducción}

La fase de contención del tratamiento de ortodoncia tiene como objetivo mantener la posición lograda de los dientes debido a la tendencia de estos a regresar a su posición original o de desplazarse a otra posición. ${ }^{1,2}$
Con este propósito, se colocan retenedores cementados los cuales pueden ser dejados a largo plazo e incluso permanentemente. ${ }^{1,3}$ Sin embargo, se han asociado los retenedores fijos a ciertos efectos negativos en la salud periodontal como el aumento en la incidencia

\footnotetext{
${ }^{1}$ Investigador independiente

${ }^{2}$ Postgrado en Ortodoncia y Ortopedia Dentomaxilofacial, Universidad de los Andes, Santiago, Chile

${ }^{\text {a }}$ Cirujano Dentista

${ }^{\mathrm{b}}$ Especialista en Ortodoncia y Ortopedia Dentomaxilofacial

${ }^{\text {c } E s p e c i a l i s t a ~ e n ~ P e r i o d o n c i a, ~ C i r u g i ́ a ~ P l a ́ s t i c a ~ P e r i o d o n t a l ~ y ~ P e r i-i m p l a n t a r i a ~}$
} 
de recesión gingival y la acumulación de placa bacteriana al dificultar una correcta higiene. ${ }^{4}$ Además, un $5 \%$ de los pacientes con retenedores fijos puede presentar movimientos indeseados, los cuales no siempre son direccionados hacia la posición pretratamiento, razón por la cual no son consideradas recidivas y en caso de no ser detectados tempranamente podrían originar alteraciones periodontales. ${ }^{5}$

Las recesiones gingivales se definen como el desplazamiento del tejido gingival marginal hacia el apical del límite amelocementario (LAC) y se clasifican dentro de las condiciones y deformidades que afectan al periodonto. ${ }^{6}$ Pueden observarse de manera localizada o generalizada e incluir una o más caras del diente. Sus principales factores desencadenantes son la inflamación asociada a placa bacteriana y el cepillado dental traumático. Otros factores son el trauma oclusal, la posición dentaria en la apófisis alveolar, la anatomía del reborde alveolar y el tabaquismo.

Se presenta un caso clínico referido a un cambio de posición dentaria asociada a una recesión gingival a pesar de tener un retenedor fijo. Los objetivos de este estudio fueron analizar las posibles razones del movimiento dentario, relacionarlo con las recesiones gingivales y analizar el momento oportuno para indicar una cirugía mucogingival.

\section{Caso clínico}

Paciente de 27 años, género femenino sin antecedentes médicos de relevancia, derivada a la especialidad de ortodoncia por mal posición de incisivo inferior asociado a recesión gingival. La paciente refirió cambio progresivo de posición posterior al término de su tratamiento ortodóncico de hace 10 años. Al examen clínico, se observó una mínima disminución de overjet y overbite, contención fija inferior de alambre trenzado cementada en incisivos y caninos, ligera giroversión de diente 1.2, torque radicular vestibular aumentado de diente 4.1 y en menor grado de 4.3 , torque radicular lingual aumentado de diente 3.1 y en menor grado de 3.2 , recesión gingival vestibular asociada a diente $4.1 \mathrm{de} 4 \mathrm{~mm}$ con ausencia de encía adherida, sin movilidad dentaria considerando la presencia de una contención lingual (Figura 1A-1B). EI diagnóstico fue de clase I esqueletal, biotipo mesofacial, leve discrepancia dentoalveolar negativa superior y clase I molar. El examen radiográfico panorámico mostró longitud radicular disminuida de segundos premolares e incisivos inferiores; este último caso, justificado por un fenómeno proyeccional (Figura 1C). El examen tomográfico muestra reabsorción ósea marginal moderada en vestibular diente $4.1 \mathrm{y}$ en lingual de 3.1 (Figura 1D).

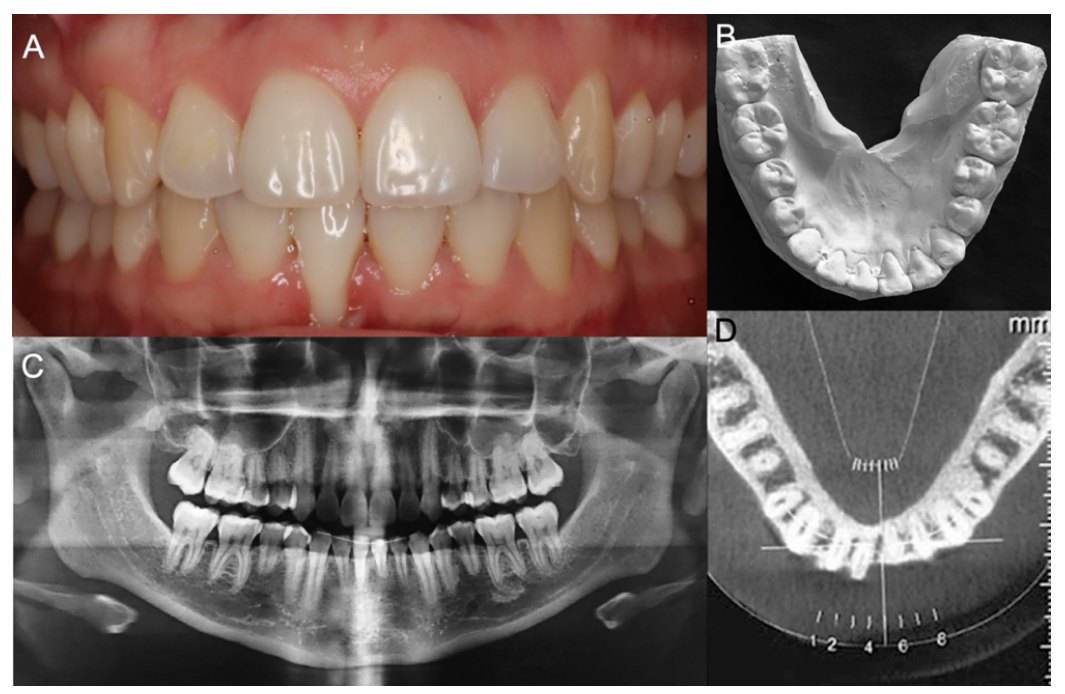

Figura 1. Fotografía y exámenes iniciales. A. Vista frontal: exposición radicular diente $4.1 \mathrm{~B}$. Modelo arcada inferior: contención fija cementada y exposición radicular diente $3.1 \mathrm{C}$. Radiografía panorámica $\mathrm{D}$. Corte transversal tomografía mandibular. 
El tratamiento consistió en dos etapas. La primera etapa implicó un retratamiento de ortodoncia para alinear, nivelar y devolver las raíces de los incisivos inferiores a sus bases óseas, y la segunda etapa involucró una cirugía periodontal para cubrir defecto por recesión.

Se inició el retratamiento con el retiro de la contención y la instalación en la arcada inferior de aparatos fijos Ispire ice de ORMCO, ranura 0,022 de prescripción MBT (McLaughlin-Bennett-Trevisi). Debido a que este caso no presentaba problemas severos de alineación, sino un torque inadecuado, se procedió con arco braided 0,017 x 0,025. Se dieron torques progresivos e individuales a este arco para manejar las posiciones radiculares.

A los siete meses, se controló mediante tomografía la relación de los incisivos inferiores con el reborde alveolar; se instalaron aparatos fijos de la misma prescripción indicada anteriormente en la arcada superior para mejor ajuste de la oclusión y la finalización del tratamiento. Se observó una disminución de la recesión vestibular de diente 4.1; sin embargo, se comenzaba a originar una en el 3.1. Por lo tanto, se realizó una interconsulta a periodoncia. A los 11 meses, se derivó a evaluación periodontal y se detectó gingivitis asociada a biofilm. La causa de esto fue la dificultad en efectuar la higiene apropiada por la ortodoncia; además, se registró que la recesión gingival vestibular del 4.1 era de 3 $\mathrm{mm}$ (Clase II de Miller) y del 3.1 de $2 \mathrm{~mm}$ (Clase I de Miller), ambas Tipo 1 en Clasificación de Cairo (Figura 2).

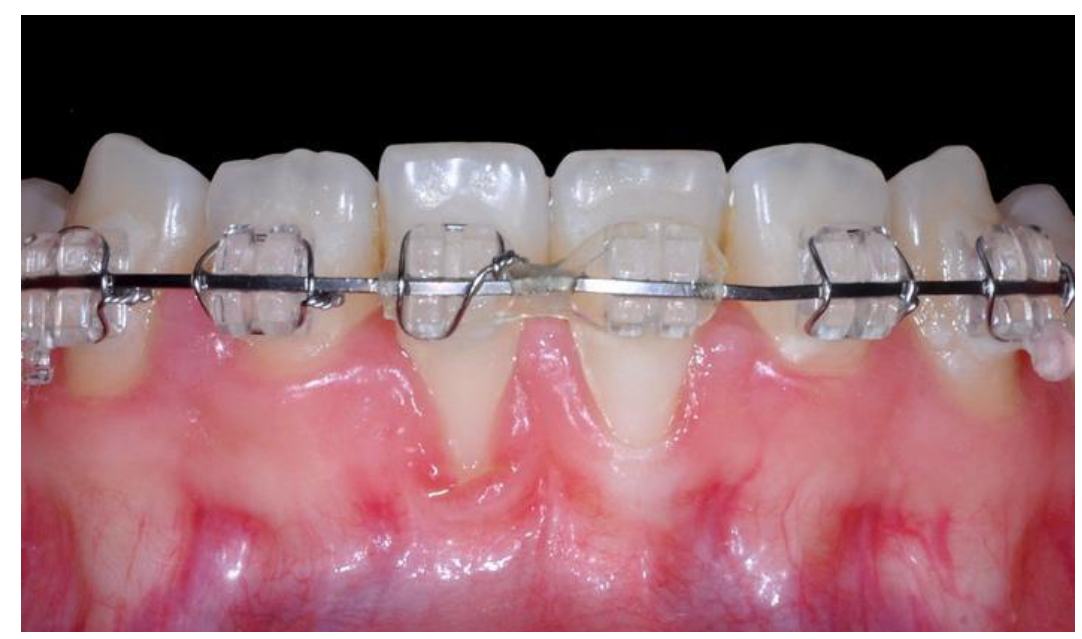

Figura 2. Fotografía evaluación periodontal.

Se solicitó una nueva tomografía de control, la cual mostró el cambio de inclinación de los incisivos con la posición deseada dentro de sus bases óseas. Para valorizar este cambio, se midió el ángulo formado entre el eje longitudinal del diente y la línea que pasa por el punto más prominente y el más anterior de la base ósea. Al comparar estas medidas con la tomografía inicial; se comprueba el cambio de torque radicular hacia lingual de diente 4.1, al disminuir ángulo; y hacia vestibular de 3.1, al aumentar ángulo. Estas observaciones, evidencian una mejora de posición y torque radicular sobre el reborde alveolar (Figura 3). 


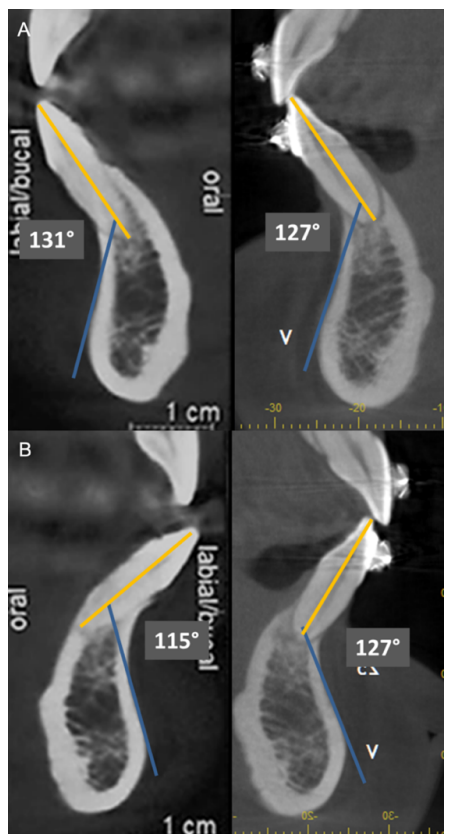

Figura 3. Comparación angulación de incisivos: tomografía inicial (lado izquierdo) y de control 11 meses (lado derecho) A. Corte sagital diente 4.1 B. Corte sagital diente 3.1.

Se decidió realizar la cirugía mucogingival después de terminar el tratamiento ortodóncico, ya que este se encontraba en la etapa final.

A los 15 meses de tratamiento, se retiraron los aparatos en ambas arcadas y se instalaron contenciones fijas superior e inferior tipo bond a braid (trenzado plano), placa Hawley superior y estampado inferior para un mejor control de los torques obtenidos (Figura 4).

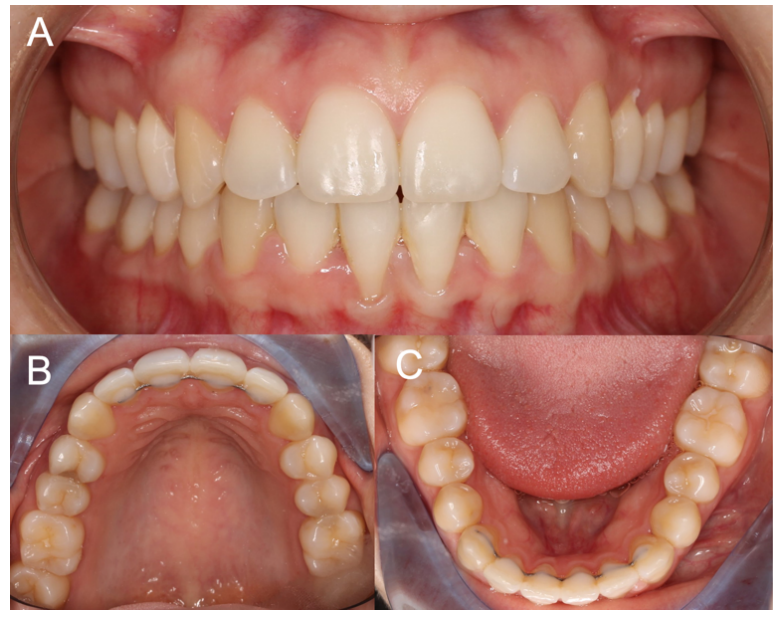

Figura 4. Fotografías al mes del retiro de aparatos ortodóncicos. A. Vista frontal B. Vista oclusal arcada superior C. Vista oclusal arcada inferior.
La cirugía mucogingival que se indicó fue la colocación de un injerto de tejido conectivo subepitelial en los dientes afectados mediante la técnica del túnel. ${ }^{7}$ Previamente a esta intervención, se le realizó un destartraje supragingival y un pulido coronario en toda la boca. La técnica quirúrgica consistió en un pulido radicular de la zona radicular expuesta de dientes 4.1 y 3.1 , así como la preparación de un túnel en la zona con un periostótomo TKN1, con la eliminación de las inserciones musculares para facilitar el desplazamiento coronal del colgajo. Luego, se tomó un injerto de tejido conectivo subepitelial del lado izquierdo del paladar con la técnica de una sola incisión de Hurzeler ${ }^{8}$ y se suturó con Vicryl 6-0 (Ethicon). Asimismo, se insertó el injerto en el túnel y una vez ajustado, se cubrió con la adaptación del colgajo $1 \mathrm{~mm}$ sobre el LAC mediante suturas ancladas a la contención lingual de politetrafluoretileno 6-0 (ADSurgical). Las suturas palatinas fueron removidas a la semana; y las demás, a las dos semanas. Se dieron indicaciones de no cepillado de la zona por 14 días y uso de colutorio de Clorhexidina $0,12 \%$ dos veces al día por dos semanas. Se dejó a la paciente con analgésico (Clonixinato de Lisina $125 \mathrm{mg}$ ) cada 6 horas. Los controles fueron efectuados a la semana 1, 2 y 8 posoperatorio (Figura 5).

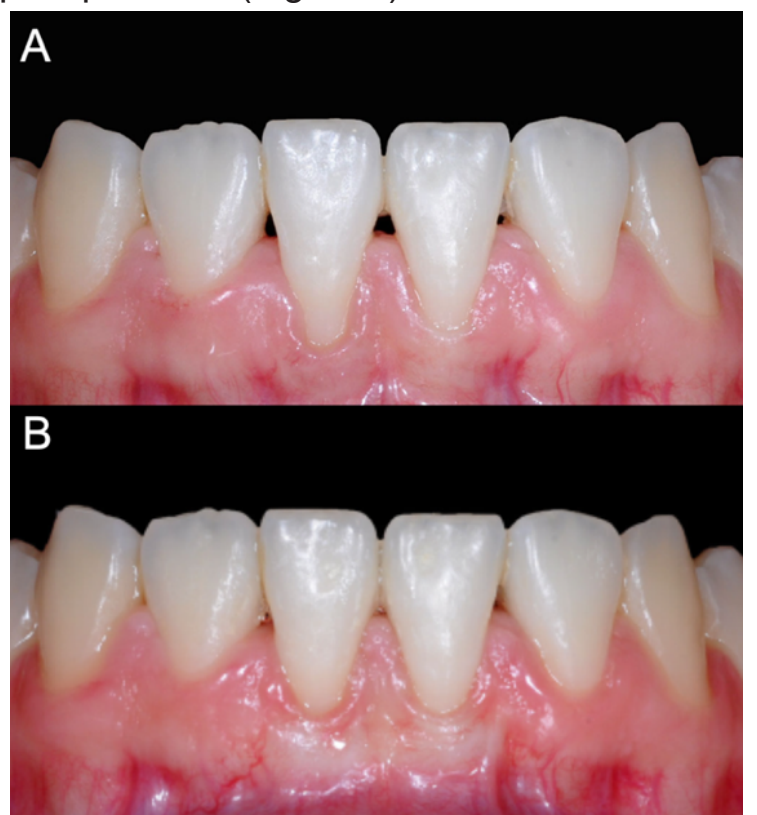

Figura 5. Evolución cirugía mucogingival. A. Posterior a ortodoncia B. Ocho semanas posteriores a cirugía. 
Una vez que la paciente estuvo estable, se aplicó el siguiente protocolo para control de las contenciones: visitas progresivas; primero, mensuales por 3 meses; luego, semestrales por un año y finalmente anuales por 5 años. Los controles periodontales son efectuados cada 6 meses (Figura 6).

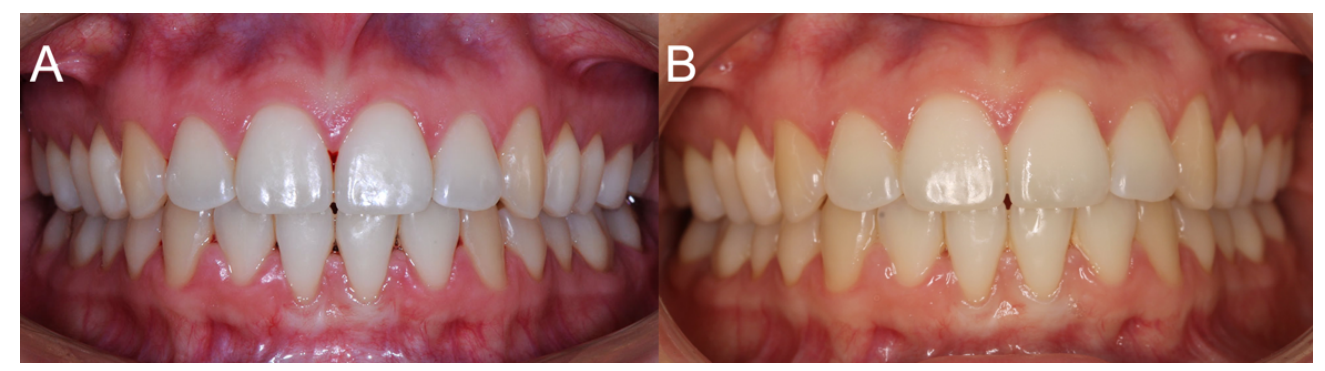

Figura 6. A. Control con periodoncista 13 meses posterior al término del tratamiento de ortodoncia y 10 meses desde cirugía. B. Control con ortodoncista 15 meses posterior al término del tratamiento de ortodoncia y 12 meses desde cirugía.

\section{Discusión}

Los movimientos inesperados de los incisivos mandibulares después del tratamiento ortodóncico pueden tener muchas causas. Estos pueden ser provocados por un componente activo del retenedor ya sea causado por el clínico durante su adaptación o cementación, o posteriormente por fuerzas masticatorias o trauma. ${ }^{9}$ La acción de la lengua está descartada, debido a que la presión que ejerce está muy por debajo de la necesaria para doblar un alambre. ${ }^{10}$ Se ha visto que el movimiento de torque ocurre con mayor probabilidad con retenedores de alambre redondo flexible de diámetro pequeño, aunque también se ha observado con retenedores de alambres flexibles de otros diámetros. ${ }^{11}$

La relación entre movimiento dentario y recesión gingival es muy discutida. Si bien el movimiento no debe considerarse como la principal causa de aparición de recesión gingival, sí puede actuar como un factor desencadenante cuando se asocia a una encía delgada, tabla ósea vestibular/lingual delgada, dehiscencias o fenestraciones óseas, presencia de inflamación o cepillado traumático. ${ }^{9}$ Concluimos que esto es lo que sucedió en este caso con la aparición de una recesión en el incisivo inferior izquierdo al ser llevado hacia vestibular durante el retratamiento. Por esto, es importante, previamente a la ortodoncia, realizar una evaluación cuidadosa del grosor bucolingual de todos los tejidos gingivales en forma individual; ya que sabemos que el fenotipo periodontal fino es más susceptible a producir recesiones que el fenotipo periodontal grueso. ${ }^{12}$ Además, el examen visual no es suficiente para determinar el grosor de los tejidos periodontales; por tanto, se sugiere complementar con Cone Beam, ya que este examen entrega una representación precisa del grosor del tejido óseo y blando cuando se usa con separadores de labios. ${ }^{13,14,15}$

Sin embargo, el movimiento dental también puede mejorar el nivel óseo marginal y la recesión gingival al redireccionar una raíz al proceso alveolar. En base esto, se planificó el tratamiento ortodóncico para una posterior cirugía. ${ }^{16}$

El momento oportuno de indicación de cirugía mucogingival para aumento gingival y cobertura radicular, debe considerar la altura de encía adherida remanente a la recesión, el grosor bucolingual del tejido gingival y la dirección del movimiento que se pretende realizar. Generalmente, en los casos donde es probable que el defecto aumente, se indica realizar la cirugía antes del tratamiento de ortodoncia; por ejemplo, cuando se realizará un movimiento hacia vestibular en un diente con dehiscencia ósea vestibular, con fenotipo gingival de riesgo (altura y espesor de encía adherida disminuída). Sin embargo, algunos recomiendan que en los casos en que se pretende favorecer la disminución del defecto al llevar el diente hacia una posición más adecuada dentro del hueso alveolar, la 
postergación de la cirugía para después del tratamiento de ortodoncia facilita el éxito del procedimiento. ${ }^{16,17}$ En este caso, se visibiliza la disminución de la recesión gingival en el diente 4.1 posterior al tratamiento de ortodoncia. Esto indica un mejor escenario para la cirugía mucogingival, pero por otra parte, se origina una recesión en el diente vecino. Por tanto, considerar una cirugía mucogingival en ambos incisivos previo a la ortodoncia para mejorar el fenotipo periodontal, podría haber sido una opción válida.

\section{Conclusión}

El control periódico de los retenedores fijos es indispensable para poder detectar en forma temprana posibles defectos en los mismos y/o cambios en la posición de los dientes. De tal forma, es posible evitar complicaciones mayores. El movimiento dentario ortodóncico puede desencadenar, agravar o mejorar una recesión gingival según la dirección del movimiento y los tejidos circundantes. Por esto, es importante una evaluación periodontal individual de cada diente antes de iniciar un tratamiento ortodóncico.

\section{Referencias}

1. Littlewood SJ, Millett DT, Doubleday B, Bearn DR, Worthington HV. Retention procedures for stabilising tooth position after treatment with orthodontic braces. Cochrane Database Syst Rev. 29 ene 2016; 2016(1):CD002283. Doi: 10.1002/14651858.CD002283.pub4.

2. Graber LW, Vanarsdall RL, Vig KWL, Huang GJ. Orthodontics: Current Principles and Techniques. 5ta ed. St. Louis, Missouri: Elsevier Health Sciences; 2012.

3. Littlewood SJ, Kandasamy S, Huang G. Retention and relapse in clinical practice. Aust Dent J. Mar 2017; 62 Suppl 1:51-57. Doi: 10.1111/adj.12475.

4. Levin L, Samorodnitzky-Naveh GR, Machtei EE. The association of orthodontic treatment and fixed retainers with gingival health. J. Periodontol. Nov 2008; 79(11):2087-2092. Doi: 10.1902/jop.2008.080128.
5. Katsaros C, Livas C, Renkema A-M. Unexpected complications of bonded mandibular lingual retainers. Am J Orthod Dentofacial Orthop. Dic 2007; $132(6): 838-41$. D o i : 10.1016/j.ajodo.2007.07.011.

6. Caton JG, Armitage G, Berglundh T, Chapple ILC, Jepsen S, Kornman KS, Mealey BL, Papapanou PN, Sanz M, Tonetti MS. A new classification scheme for periodontal and peri-implant diseases and conditions - Introduction and key changes from the 1999 classification. J Clin Periodontol. Jun 2018; 45 Suppl 20:S1-S8. Doi: 10.1111/jcpe.12935..

7. Azzi R, Etienne D. Root coverage and papilla reconstruction by connective tissue graft inserted under a vestibular coronally advanced tunnelized flap. J Periodont Implant Orale. 1998;17: 71-7.

8. Hurzeler MB, Weng D. A single- incision technique to harvest subepithelial connective tissue grafts from the palate. Int J Periodontics Restor Dent. Jun 1999;19(3): 279-87. PMID: 10635174.

9. Taffarel IP, Miyoshi C S, Maruo I T, Meira TM, Tanaka OM. The Periodontal Benefit of Orthodontic Tooth Movement in a Deep Facial Recession of a Mandibular Incisor. Case Rep Dent. 2019; 2019:1-6. Doi: $10.1155 / 2019 / 7601475$

10. Proffit WR. Muscle pressures and tooth position: North American whites and Australian aborigines. Angle Orthod. Ene 1975; 45(1):1-11. Doi: 10.1043/00033219(1975)045<0001:MPATPN>2.0.C $0 ; 2$.

11. Shaughnessy TG, Proffit WR, Samara SA. Inadvertent tooth movement with fixed lingual retainers. Am $J$ Orthod De ntofacial Orthop. Fe b $2016 ; 149: 277-86$. D o i : 10.1016/j.ajodo.2015.10.015.

12. Olsson M, Lindhe J. Periodontal characteristics in individuals with varying form of the upper central incisors. J Clin Peridontol. Ene 1991;18(1): 78-82. Doi: 10.1111/j.1600051x.1991.tb01124.x.

13. Eghbali $A$, De Rouck $T$, De Bruyn $H$, Cosyn J. The gingival biotype assessed by experienced and inexperienced 
clinicians. J Clin Periodontol. Nov 2009; 36:958-63. Doi: 10.1111/j.1600051X.2009.01479.x. Epub 2009 Oct 6. PMID: 19811580.

14. Fu J-H, Yeh C-Y, Chan H-L, Tatarakis N, Leong DJM, Wang H-L. Tissue biotype and its relation to the underlying bone morphology. J Periodontol. 2010; 81(4):569-74. Fu JH, Yeh CY, Chan HL, Tatarakis N, Leong DJ, Wang HL. Tissue biotype and its relation to the underlying bone morphology. J Periodontol. Apr 2010 ; 81 (4):569-74. Doi : 10.1902/jop.2009.090591.

15. Januário $A L$, Barriviera $M$, Duarte WR. Soft tissue cone-beam computed tomography: a novel method for the measurement of gingival tissue and the dimensions of the dentogingival unit. $J$ Esthet Restor Dent. 2008; 20(6):366-73; discussion 374 . Doi: 10.1111/j.17088240.2008.00210.x.

16. Laursen MG, Rylev M, Melsen B. The role of orthodontics in the repair of gingival recessions. Am J Orthod Dentofacial Orthop. Ene 2020;157(1): $29-34$. D o i : 10.1016/j.ajodo.2019.01.023.

17. Wennstrb JL. Mucogingival Considerations in Orthodontic Treatment. Semin Orthod. 1996; 2(1):46-54. Doi: 10.1016/S10738746(96)80039-9.
Correspondencia:

constanzaurtubiam@gmail.com
Fecha de recepción : :18 de junio de 2020

Fecha de aceptación : 01 de agosto de 2020 